\title{
Deep Learning for Automated Recognition of Covid-19 from Chest X-ray Images
}

\author{
Linh T. Duong ${ }^{\mathrm{a}}$, Phuong T. Nguyen ${ }^{\mathrm{b}, *}$, Ludovico Iovino ${ }^{\mathrm{c}}$, Michele Flammini $^{\mathrm{c}}$ \\ ${ }^{a}$ Institute of Research and Development, Duy Tan University, Vietnam \\ duongtuanlinh@duytan.edu.vn \\ ${ }^{b}$ Department of Information Engineering, Computer Science and Mathematics \\ University of L'Aquila, Italy \\ phuong.nguyen@univaq.it \\ ${ }^{c}$ Gran Sasso Science Institute, Italy \\ \{ludovico.iovino, michele.flammini\}@gssi.it
}

\begin{abstract}
Background: The pandemic caused by coronavirus in recent months is having a devastating global effect, which puts the world under the most ever unprecedented emergency. Currently, since there are not effective antiviral treatments for Covid-19 yet, it is crucial to early detect and monitor the progression of the disease, thus helping to reduce mortality. While a corresponding vaccine is being developed, and different measures are being used to combat the virus, medical imaging techniques have also been investigated to assist doctors in diagnosing this disease. Objective: This paper presents a practical solution for the detection of Covid-19 from chest X-ray (CXR) images, exploiting cutting-edge Machine Learning techniques. Methods: We employ EfficientNet and MixNet, two recently developed families of deep neural networks, as the main classification engine. Furthermore, we also apply different transfer learning strategies, aiming at making the training process more accurate and efficient. The proposed approach has been validated by means of two real datasets, the former consists of 13,511 training images and 1,489 testing images, the latter has 14,324 and 3,581 images for training and testing, respectively. Results: The results are promising: by all the experimental configurations considered in the evaluation,
\end{abstract}

\footnotetext{
${ }^{*}$ Corresponding author
} 
our approach always yields an accuracy larger than 95.0\%, with the maximum accuracy obtained being $96.64 \%$. Conclusions: As a comparison with various existing studies, we can thus conclude that our performance improvement is significant.

\section{Introduction}

Covid-19 is a coronavirus-induced infection that can be associated with a coagulopathy and infection-induced inflammatory changes [1]. The disease poses a serious threat to public health, and thus in March 2020, the World Health Or5 ganization (WHO) declared Covid-19 a pandemic. So far, the virus has infected more than ten millions of people across the world, and has claimed over five hundreds thousands peoples' lives. The clinical spectrum of the disease is very wide, ranging from fever, dry cough and diarrhea, but can be combined with mild pneumonia and mild dyspnoea. In some cases, the infection can evolve to severe pneumonia, causing approximately $5 \%$ of the infected patients to severe lung dysfunction. Given the circumstances, patients need ventilation as they are highly exposed to multiple extra pulmonary organ failure.

Since so far there have been no effective antiviral vaccines for Covid-19, it is crucial to reduce mortality by early detecting and monitoring the progression of the disease [2], so as to effectively personalize patient's treatment. Radiology is part of a fundamental process to detect whether or not the radiological outcomes are consistent with the infection and radiologists should expedite as much as possible the exploration, and provide accurate reports of their findings. Chest X-ray (CXR) images of Covid-19 patients usually show multifocal, bilateral and

2o peripheral lesions, but in the early phase of the disease they may present a unifocal lesion, most commonly located in the inferior lobe of the right lung. Providing doctors with a preliminary diagnosis of Covid-19 from CXR images would be of great importance, also considering the number of false positives obtained by swab results. 
In recent years, Artificial Intelligence (AI) has been in the forefront of methodologies applied to improve products and services in various aspects of everyday life. The proliferation of advanced Machine Learning algorithms enables a numerous number of applications in various domains. Machine Learning (ML) algorithms attempt to simulate humans' cognitive functions [3], aiming to acquire real-world knowledge autonomously [4]. In this way, ML techniques are capable of conceptualizing from concrete examples, without needing to be manually coded $[5,6]$. Thanks to this characteristic, they have applications in various domains. For example, they have been applied to improve Web search by learning from a user's long-term search history [7]. For recommender systems, ML algorithms demonstrate their superiority by analyzing sentiment with ensemble techniques in social applications [8], or allowing systems to learn from various profiles, thus boosting up the recommendation outcomes [4]. In the Health care sector, the potential of ML to allow for rapid diagnosis of diseases has also been proven by various research work [3, 9, 10, 11].

Aiming to assist the clinical care, this paper presents a practical solution for the detection of Covid-19 from CXR images exploiting two cutting-edge deep neural network families, EfficientNet [12] and MixNet [13], empowering the learning process by means of three different transfer learning strategies, namely ImageNet [14], AdvProp [15], and Noisy Student [16]. Our ex${ }_{45}$ perimental results on two considerably large datasets show that the proposed solution outperforms the existing studies that we are aware of, in terms prediction accuracy. The main contributions of our work can thus be summarized as follows:

- A framework for recognition of Covid-19 from CXR images using state-ofthe-art deep learning techniques;

- A successful empirical evaluation on two large datasets of CXR images;

- A software prototype in the form of a mobile app ready to be downloaded. 
The paper is organized into the following sections. In Section 2 we briefly review convolutional neural networks, EfficientNet and MixNet as well as the transfer learning methods. Section 3 explains the dataset and metrics used for our evaluation, together with the main results. The related work is reviewed in Section 5. Finally, Section 6 provides some conclusive remarks and discusses possible future research directions.

\section{Background}

As a base for our presentation, we provide a background on convolutional neural networks in Section 2.1. Two families of deep neural networks, i.e., EfficientNet and MixNet, which are used as the classification engine in our work, are introduced in Section 2.2. Finally, a brief introduction to transfer learning is given in Section 2.3. \\ 2.1. Fundamentals to convolutional neural networks}

Convolutional neural networks (CNNs) [17] are a family of supervised learning techniques that work on images, attempting to capture some of their intrinsic features, such as spatial and temporal structures, using a filter or kernel. A filter is a small square sliding window, and it is used to capture features from an input image, such as nodes and edges. Various types of features can be captured with several filters. The convolution operation is performed by sliding the filter along the width and height of a feature map, which is either the input image, or the result of the convolution operation. An output feature map of one layer becomes the input feature map of the succeeding layer. In general, a CNN also contains the following intrinsic elements:

$\triangleright$ Convolution layer: as the name suggests, this layer extracts important features of an input image by convolving the image with filters;

$\triangleright$ Pooling layer: such a layer is used to downsample a feature map by taking the maximum value within a window, normally a square one, to reduce the number of parameters [17]; 


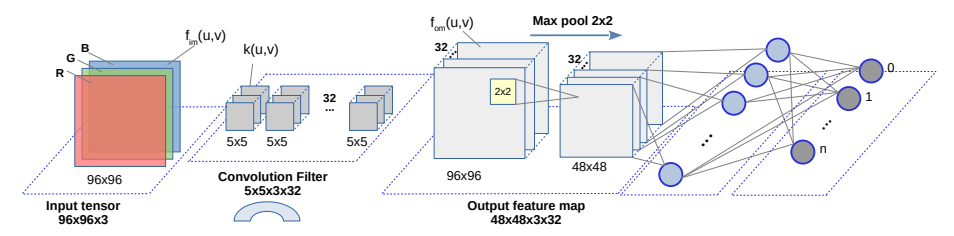

Figure 1: Filtering and pooling.

$\triangleright$ Fully-connected layer: the layer works as a conventional perceptron, each of its neurons is fully connected to the previous layer.

$\triangleright$ Dropout: it is used to distribute the learned representation across all the neurons. Dropout is an effective measure to combat overfitting [18];

$85 \triangleright$ Softmax: the function converts a set of real numbers to probabilities, which in turn sum to 1.0 [17]. Softmax is normally used as activation function in the last fully-connected layer of a CNN. Given C categories, denoted as $y_{k}$ the output of the $\mathrm{k}^{\text {th }}$ neuron, the class that gets the maximum probability is selected as the final prediction, i.e., $\hat{y}=\operatorname{argmax} p_{k}, k \in 1 . . C$, with $p_{k}$ being defined as below.

$$
p_{k}=\frac{\exp \left(y_{k}\right)}{\sum_{k=1}^{C} \exp \left(y_{k}\right)}
$$

$\triangleright$ Rectified Linear Units (ReLU): convolutional layers use ReLU as the activation function, which returns 0 given a negative input, and returns the input itself if it is larger than 0 , i.e., $f(x)=\max (0, x)$.

Figure 1 illustrates typical convolution and pooling operations in image classification. A tensor of size $96 \times 96 \times 3$ represents an input image, where $96 \times 96$ 95 is the image size, while the number 3 corresponds to three color channels in images, i.e., Red (R), Green (G), and Blue (B). The convolution operation is performed where a $4 \mathrm{D}$ filter of size $5 \times 5 \times 3 \times 32$ is convolved with the input feature map to produce an output feature map of size $96 \times 96 \times 1 \times 32$. To illustrate how a CNN works, we consider only a slice of the $4 \mathrm{D}$ filter, corresponding to a matrix $k(u, v)$. Pooling is done by means of a Maxpool $2 \times 2$ element for reducing the resulted feature map's width and height of a half, culminating in an output feature map of size $48 \times 48 \times 1 \times 32$. 


\subsection{EfficientNet and MixNet}

Based on the observation that a better accuracy and efficiency can be ob-

to the EfficientNet family, other configurations of the MixNet family, such as MixNet-Medium or MixNet-Large, are derived from MixNet-S with different scaling values. 


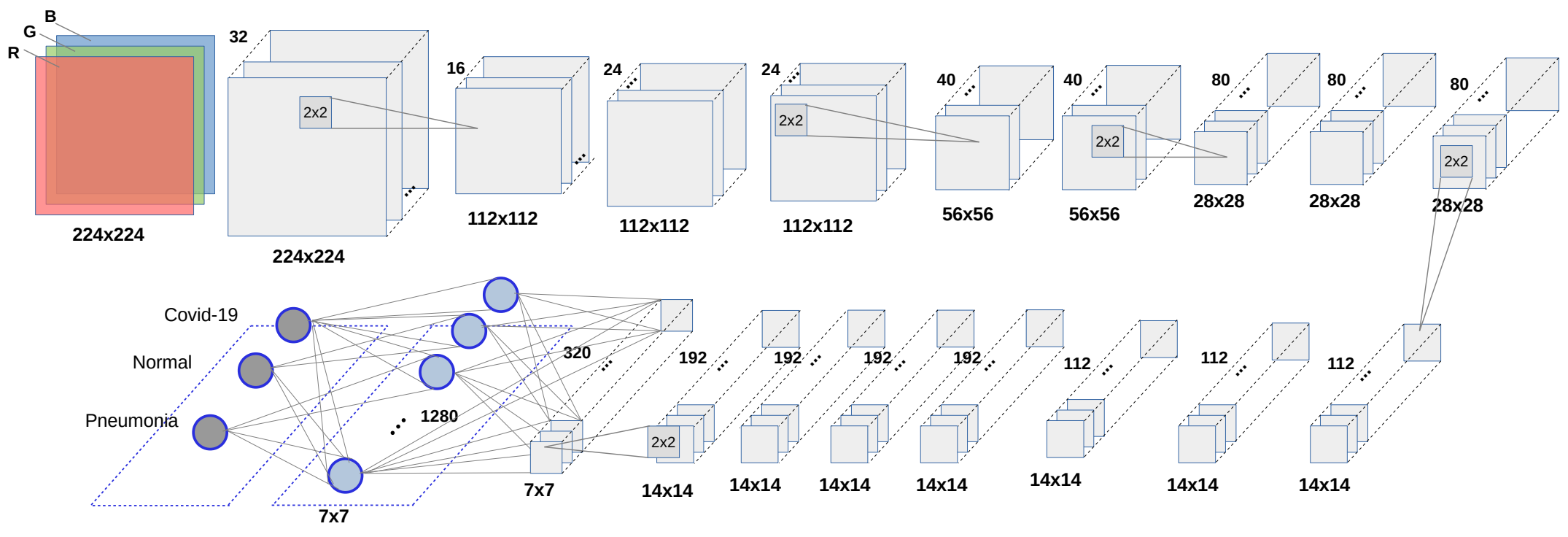

Figure 2: EfficientNet-B0 architecture. 


\subsection{Transfer learning}

In order to tune their internal parameters, i.e., weights and biases, normally CNNs need a huge amount of labeled data. Furthermore, the deeper a network is, the more parameters it contains. In this respect, deeper networks would require more data to prevent overfitting and be effective. As a result, it is crucial to feed them with enough data, so as to foster the training process. However, such a requirement is hard to be met in practice, since the labeling process usually is made manually, thus being time consuming and prone to error [25]. To this end, transfer learning has been conceptualized as an effective way to extract and transfer the knowledge from a well-defined source domain to a novice target domain $[26,27]$. In other words, transfer learning facilitates the export of existing convolution weights from a model trained using large datasets to create new accurate models exploiting a relatively lower number of labeled images. As it has been shown in various studies [28, 29], transfer learning remains helpful even when the target domain is quite different from the one in which the original weights have been obtained. In this work, we consider the following learning methods:

- ImageNet [14]: The ImageNet dataset has been widely exploited to apply transfer learning by several studies, since it contains more than 14 million images, covering miscellaneous categories;

- AdvProp [15]: adversarial propagation has been proposed as an improved training scheme, with the ultimate aim of avoiding overfitting. The method treats adversarial examples as additional examples, and uses a separate auxiliary batch norm for adversarial examples;

- NS [16]: the Noisy Student learning method attempts to improve ImageNet classification Noisy Student Training by: (i) enlarging the trainee/student equal to or larger than the trainer/teacher, aiming to make the trainee learn better on a large dataset, and (ii) adding noise to the student, thus forcing him to learn more. 
In an attempt to develop an expert system that can help doctors to early detect Covid-19 from CXR images, we make use of EfficientNet and MixNet as the classification engine. Moreover, we obtain network weights by means of the three different learning strategies mentioned above, i.e., ImageNet, AdvProp, and NS. In the following section, we present the evaluation settings used to study the performance of our approach.

\section{Evaluation}

This section explains in detail the material and methods used to evaluate the proposed approach. In particular, we made use of two existing datasets and recent implementations ${ }^{1}$ of EfficientNet and MixNet, which were built on top of the PyTorch framework. ${ }^{2}$ Moreover we adopted pre-trained weights from different sources to speed up the learning process. The tool developed through this paper has been also published in GitHub to make it available for future research. ${ }^{3}$

\subsection{Research questions}

We answer the following research questions to study the performance of the classifiers with respect to the different transfer learning methods:

- $\mathbf{R Q}_{1}$ : Which network family between EfficientNet and MixNet brings the best prediction performance? For a classifier, it is crucial to get accurate outcomes, according to various quality metrics. We determine which deep neural network family yields the best prediction performance.

- $\mathbf{R Q}_{2}$ : Which transfer learning technique is beneficial to the final outcome? We are interested in finding which transfer learning method between ImageNet, AdvProp, and NS helps which network, i.e., EfficientNet and MixNet, to obtain a better outcome.

\footnotetext{
${ }^{1}$ https://github.com/rwightman/gen-efficientnet-pytorch

${ }^{2}$ https://pytorch.org

${ }^{3}$ https://github.com/linhduongtuan/Covid-19_Xray_Classifier/
} 


\subsection{Datasets}

We exploited existing datasets, used by some preovious works [30, 31], to study the performance of our approach. Their characteristics are summarized in Table 1. In each dataset there are three categories, i.e., Covid-19, Normal, and Pneumonia. While there is only a category with no symptom, i.e., Normal, the other two categories, Covid-19 and Pneumonia, represent different levels of infection-induced inflammatory changes. Dataset $\mathbf{D}_{1}$ consists of 13,511 images for training and 1,489 images for testing. We see that it has an imbalance among the categories, as there is a large number of images for Normal and Pneumonia, but only 108 ones for Covid-19. $\mathbf{D}_{1}$ is used as a means to compare our approach with existing studies that performed validation on the same dataset. In particular, by exploiting $\mathbf{D}_{1}$ we attempt to compare the performance of our approach with that of other studies that exploited $\mathbf{D}_{1}$ in their evaluation [30, 31]. Meanwhile, $\mathbf{D}_{2}$ is newly updated with more data for training and testing. There are some overlaps between $\mathbf{D}_{1}$ and $\mathbf{D}_{2}: \mathbf{D}_{2}$ is actually an extension of $\mathbf{D}_{1}$ with some addition and removal, here and there. In particular, $\mathbf{D}_{2}$ consists of 14,324 and 3,581 images for training and testing, respectively. We made use of $\mathbf{D}_{2}$ to validate the performance of our approach on a larger amount of data, showing its applicability in practice.

\begin{tabular}{|l|l|l|l|l|l|}
\hline \multirow{2}{*}{ Dataset } & \multirow{2}{*}{ Type } & \multicolumn{3}{|c|}{ Categories } & \multirow{2}{*}{ Total } \\
\cline { 3 - 6 } & & Covid-19 & Normal & Pneumonia & \\
\hline \hline \multirow{3}{*}{$\mathbf{D}_{1}$} & Train & 98 & 7,966 & 5,447 & 13,511 \\
\cline { 2 - 6 } & Test & 10 & 885 & 594 & 1,489 \\
\cline { 2 - 6 } & Total & $\mathbf{1 0 8}$ & $\mathbf{8 , 8 5 1}$ & $\mathbf{6 , 0 4 1}$ & $\mathbf{1 5 , 0 0 0}$ \\
\hline \hline \multirow{3}{*}{$\mathbf{D}_{2}$} & Train & 261 & 8,154 & 5,909 & 14,324 \\
\cline { 2 - 6 } & Test & 66 & 2,038 & 1,477 & 3,581 \\
\cline { 2 - 6 } & Total & $\mathbf{3 2 7}$ & $\mathbf{1 0 , 1 9 2}$ & $\mathbf{7 , 3 8 6}$ & $\mathbf{1 7 , 9 0 5}$ \\
\hline
\end{tabular}

Table 1: Datasets. 


\subsection{Evaluation Metrics}

All images in $\mathbf{D}_{1}$ and $\mathbf{D}_{2}$ have been assigned a label, i.e., either Normal or Pneumonia or Covid-19. From the testing data, three independent groups

Precision and Recall: Precision measures the ratio of classified images the number of true positives found in the ground-truth data.

$$
\text { precision }_{i}=\frac{T P_{i}}{\left|C_{i}\right|} \quad \text { (3) } \quad \text { recall }_{i}=\frac{T P_{i}}{\left|G_{i}\right|}
$$

$\mathbf{F}_{1}$ score (F-Measure): The metric is computed as the harmonic average of precision and recall by means of the following formula:

$$
F_{1}=\frac{2 \cdot \text { precision }_{i} \cdot \text { recall }_{i}}{\text { precision }_{i}+\text { recall }_{i}}
$$

Furthermore, we make use of an additional metric to measure the computational efficiency. 
Recognition speed: We measure the average number of generated predictions per second, using a system whose configurations are presented in Table 2.

\subsection{Settings}

To train deep neural networks such as EfficientNet and MixNet, it is necessary to have a server with a powerful computational capability. Table 2 specifies the hardware and software configurations of the system used to conduct our study.

\begin{tabular}{|l|l|}
\hline Name & Description \\
\hline \hline RAM & $24 \mathrm{~GB}$ \\
\hline CPU & Intel@ Core ${ }^{\mathrm{TM}}$ i5-2400 CPU @ $3.10 \mathrm{GHz} \times 4$ \\
\hline GPU & GeForce GTX $1080 \mathrm{Ti}$ \\
\hline OS & Ubuntu 18.04 \\
\hline Python & 3.7 .5 \\
\hline Pytorch & 1.5 \\
\hline Torchvision & 0.5 .0 \\
\hline Numpy & 1.15 .4 \\
\hline Git & 2.0 \\
\hline Timm & 0.1 .26 \\
\hline
\end{tabular}

Table 2: Hardware and software configurations.

\begin{tabular}{|l|l|l|l|l|l|}
\hline Conf & Network & Batch size & \# of Params & Learning Method & Size (MB) \\
\hline \hline $\mathrm{C}_{1}$ & EfficientNet-B0 & 110 & $7,919,391$ & ImageNet & 53.1 \\
\hline $\mathrm{C}_{2}$ & EfficientNet-B0 & 110 & $7,919,391$ & AdvProp & 53.1 \\
\hline $\mathrm{C}_{3}$ & EfficientNet-B0 & 110 & $7,919,391$ & NS & 53.1 \\
\hline $\mathrm{C}_{4}$ & EfficientNet-B3 & 64 & $\mathbf{1 4 , 3 5 2 , 0 7 5}$ & ImageNet & $\mathbf{1 0 6 . 9}$ \\
\hline $\mathrm{C}_{5}$ & EfficientNet-B3 & 64 & $\mathbf{1 4 , 3 5 2 , 0 7 5}$ & AdvProp & $\mathbf{1 0 6 . 9}$ \\
\hline $\mathrm{C}_{6}$ & EfficientNet-B3 & 64 & $\mathbf{1 4 , 3 5 2 , 0 7 5}$ & NS & $\mathbf{1 0 6 . 9}$ \\
\hline \hline $\mathrm{C}_{7}$ & MixNet-Small & 110 & $6,253,449$ & ImageNet & 41.8 \\
\hline $\mathrm{C}_{8}$ & MixNet-Medium & 90 & $7,133,225$ & ImageNet & 48.9 \\
\hline $\mathrm{C}_{9}$ & MixNet-Large & 60 & $9,448,095$ & ImageNet & 67.5 \\
\hline $\mathrm{C}_{10}$ & MixNet-XL & 60 & $14,015,611$ & ImageNet & 104.2 \\
\hline
\end{tabular}

Table 3: Experimental configurations.

We consider two network families with the learning strategies mentioned in Section 2.3, resulting in ten independent configurations, i.e., $\mathrm{C}_{i}, \mathrm{i}=1, . ., 10$. 
Concerning the EfficientNet family, through our empirical study we realized that two configurations, EfficientNet-B0 and EfficientNet-B3, are more effective than the others on the considered datasets, and thus we selected them for our evaluation. For the MixNet family, we made use of four different configurations, i.e., MixNet-Small, MixNet-Medium, MixNet-Large and MixNet-XL. It is worth mentioning that for the MixNet family there are only weights coming from the ImageNet dataset available, while for the EfficientNet one we obtained weights for all the transfer learning techniques mentioned in Section 2.3. The ten different experimental configurations are explained in Table 3. The Batch size column specifies the number of items used for each training step; \# of Params is the number of parameters used by each network; and finally Size corresponds to the file size needed to store the parameters. It is clear that EfficientNet-B3 is the largest network with respect to the number of parameters as well as the file size to store them. In particular, all the EfficientNet-B3 configurations, i.e., $\mathrm{C}_{4}$, $\mathrm{C}_{5}$, and $\mathrm{C}_{6}$ need more than 14 millions of parameters, accounting for more than $100 \mathrm{MB}$ of storage space each. In the evaluation, we applied the five-fold cross validation technique on the datasets, i.e., each dataset is divided into five equal parts and each validation was performed in five independent rounds. By each round, one part is used as testing and the other four parts are used as training.

In the next section, we present in detail the experimental results by referring to the aforementioned research questions.

\section{Experimental Results}

This section reports and analyzes the results obtained from our experiments. We address our two research questions separately. best prediction performance?

Table 4 reports the results we obtained by performing the experiments on dataset $\mathbf{D}_{1}$. In all the network configurations, the corresponding accuracy is 
always larger than $95 \%$. The maximum accuracy is $96.64 \%$, and is obtained

270

vProp. Concerning Precision, almost all the configurations get 1.000 as precision for the Covid-19 category. This means that all images classified as Covid19 by the classifiers are actually Covid-19. For the other two categories, i.e., Normal and Pneumonia, the maximum precision is 0.968 , achieved by $\mathbf{C}_{5}$ for

275 Category Normal, and by $\mathbf{C}_{4}$ for Category Pneumonia. Overall, we see that all the classifiers are able to predict the testing images with high precision.

With respect to recall, we can see that for category Covid-19, all the classifiers get a considerably low score. In particular, the highest recall is 0.700 , obtained by $\mathbf{C}_{5}$. This means that while the approach is able to find good predictions for the category, it cannot return all the items in the ground-truth data. We suppose that this happens due to the limited data available for training. As shown in Table 1, with the Covid-19 category there are only 98 images and 10 images for training and testing, respectively. Meanwhile, for other two image categories, the recall scores are substantially improved. The best recall is seen by category Normal, i.e., 0.985 ; while by Pneumonia, recall is 0.952 . As we see in Table 1, both categories have a larger number of training and testing images compared to the Covid-19 category. 


\begin{tabular}{|l|l|l|l|l|l|l|l|l|l|l|}
\hline Configuration & Accuracy & \multicolumn{3}{|c|}{ Precision } & \multicolumn{3}{c|}{ Recall } & \multicolumn{3}{c|}{ F $_{1-s c o r e}$} \\
\hline- & - & Covid-19 & Normal & Pneu. & Covid-19 & Normal & Pneu. & Covid-19 & Normal & Pneu. \\
\hline \hline $\mathrm{C}_{1}$ & $95.64 \%$ & $\mathbf{1 . 0 0 0}$ & 0.952 & 0.961 & 0.300 & 0.981 & 0.929 & 0.461 & 0.967 & 0.945 \\
\hline $\mathrm{C}_{2}$ & $95.77 \%$ & $\mathbf{1 . 0 0 0}$ & 0.960 & 0.954 & 0.300 & 0.975 & 0.942 & 0.461 & 0.967 & 0.948 \\
\hline $\mathrm{C}_{3}$ & $95.30 \%$ & $\mathbf{1 . 0 0 0}$ & 0.950 & 0.956 & 0.300 & 0.977 & 0.927 & 0.461 & 0.963 & 0.941 \\
\hline $\mathrm{C}_{4}$ & $96.17 \%$ & $\mathbf{1 . 0 0 0}$ & 0.957 & $\mathbf{0 . 9 6 8}$ & 0.300 & $\mathbf{0 . 9 8 5}$ & 0.937 & 0.461 & 0.971 & 0.953 \\
\hline $\mathrm{C}_{5}$ & $\mathbf{9 6 . 6 4 \%}$ & 0.875 & $\mathbf{0 . 9 6 8}$ & 0.964 & $\mathbf{0 . 7 0 0}$ & 0.978 & $\mathbf{0 . 9 5 2}$ & $\mathbf{0 . 7 7 8}$ & $\mathbf{0 . 9 7 3}$ & $\mathbf{0 . 9 5 8}$ \\
\hline $\mathrm{C}_{6}$ & $95.90 \%$ & 0.857 & 0.957 & 0.963 & 0.600 & 0.978 & 0.936 & 0.705 & 0.967 & 0.949 \\
\hline \hline $\mathrm{C}_{7}$ & $95.30 \%$ & $\mathbf{1 . 0 0 0}$ & 0.953 & 0.951 & 0.300 & 0.974 & 0.932 & 0.461 & 0.963 & 0.942 \\
\hline $\mathrm{C}_{8}$ & $95.98 \%$ & $\mathbf{1 . 0 0 0}$ & 0.966 & 0.950 & 0.400 & 0.971 & 0.951 & 0.571 & 0.969 & 0.954 \\
\hline $\mathrm{C}_{9}$ & $96.11 \%$ & $\mathbf{1 . 0 0 0}$ & 0.961 & 0.960 & 0.400 & 0.978 & 0.944 & 0.571 & 0.969 & 0.952 \\
\hline $\mathrm{C}_{10}$ & $96.37 \%$ & $\mathbf{1 . 0 0 0}$ & 0.964 & 0.962 & 0.600 & 0.978 & 0.947 & 0.750 & 0.971 & 0.955 \\
\hline
\end{tabular}

Table 4: Experimental results on dataset $\mathbf{D}_{1}$.

\begin{tabular}{|l|l|l|l|l|l|l|l|l|l|l|l|}
\hline Configuration & Accuracy & \multicolumn{3}{|c|}{ Precision } & \multicolumn{3}{c|}{ Recall } & \multicolumn{3}{c|}{ F $_{1}$-score } \\
\hline- & - & Covid-19 & Normal & Pneu. & Covid-19 & Normal & Pneu. & Covid-19 & Normal & Pneu. \\
\hline \hline $\mathrm{C}_{1}$ & $\mathbf{9 5 . 8 1 \%}$ & 0.968 & $\mathbf{0 . 9 5 8}$ & 0.957 & $\mathbf{0 . 9 2 4}$ & 0.970 & $\mathbf{0 . 9 4 2}$ & $\mathbf{0 . 9 4 5}$ & 0.644 & $\mathbf{0 . 9 5 0}$ \\
\hline $\mathrm{C}_{2}$ & $94.39 \%$ & 0.948 & 0.942 & 0.946 & 0.560 & 0.972 & 0.922 & 0.704 & 0.956 & 0.934 \\
\hline $\mathrm{C}_{3}$ & $93.30 \%$ & 0.889 & 0.932 & 0.935 & 0.363 & 0.971 & 0.906 & 0.616 & 0.951 & 0.920 \\
\hline $\mathrm{C}_{4}$ & $95.05 \%$ & 0.950 & 0.942 & $\mathbf{0 . 9 6 4}$ & 0.863 & $\mathbf{0 . 9 7 7}$ & 0.912 & 0.905 & 0.959 & 0.940 \\
\hline $\mathrm{C}_{5}$ & $95.59 \%$ & 0.968 & 0.955 & 0.955 & 0.924 & 0.968 & 0.939 & 0.945 & $\mathbf{0 . 9 6 2}$ & 0.947 \\
\hline $\mathrm{C}_{6}$ & $95.00 \%$ & $\mathbf{0 . 9 7 8}$ & 0.953 & 0.944 & 0.667 & 0.968 & 0.937 & 0.792 & 0.960 & 0.940 \\
\hline \hline $\mathrm{C}_{10}$ & $95.53 \%$ & 0.967 & 0.951 & 0.960 & 0.909 & 0.973 & 0.932 & 0.937 & $\mathbf{0 . 9 6 2}$ & 0.946 \\
\hline
\end{tabular}

Table 5: Experimental results on dataset $\mathbf{D}_{2}$. 
Concerning the $F_{1}$ scores, we see that for the Covid-19 category, the maximum $F_{1}$ is 0.778 , obtained by $\mathrm{C}_{5}$. Meanwhile, by the other configurations, maximum accuracy is $93.0 \%$ with similar experimental settings. In this respect, we conclude that application of the two network families EfficientNet and MixNet as well as the different transfer learning techniques brings a good prediction performance on the considered dataset.

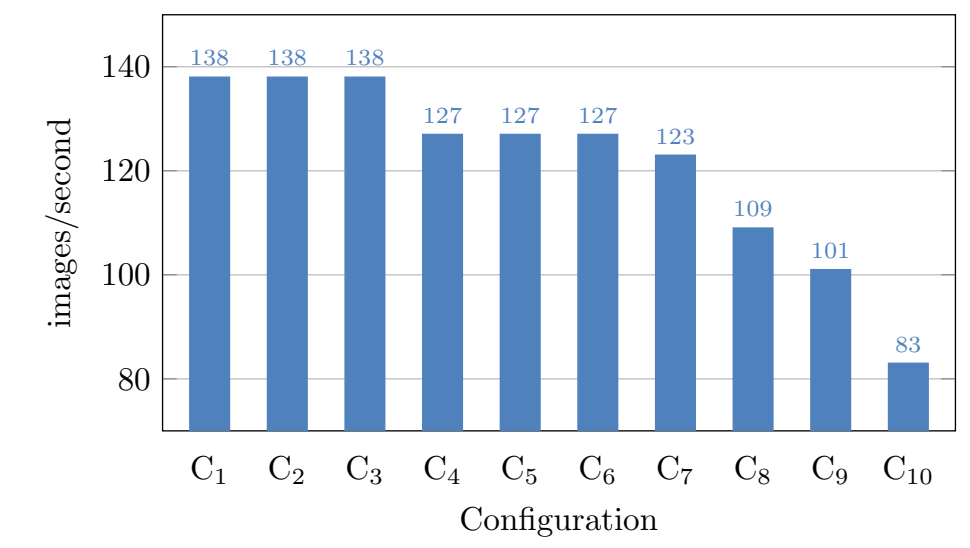

Figure 3: Recognition speed for the configurations on dataset $\mathrm{D}_{1}$. as shown above. For the other two categories Normal and Pneumonia, the $F_{1}$ scores are improved considerably compared to Covid-19. It is evident that $\mathrm{C}_{5}$ obtains the best $F_{1}$ scores for all the three categories, i.e., also 0.973 for Normal and 0.958 for Pneumonia.

with the gray color, is the configuration among the others that brings the best prediction performance.

Compared to existing work that performs evaluation on the same dataset [30, 32], our approach achieves a better performance with respect to accuracy, precision, recall, and $\mathrm{F}_{1}$-score. For instance, the work by Wang et al. [30], the

Using the system specified in Table 3, we counted the number of predictions returned by the classifiers in a second, as depicted in Fig. 3. From the figure it 
is clear that $\mathrm{C}_{1}, \mathrm{C}_{2}$, and $\mathrm{C}_{3}$, corresponding to using EfficientNet-B0 as classification engine, are the most efficient configurations, as they return 138 images per second in average. EfficientNet-B3 also yields a good timing performance, i.e., using $\mathrm{C}_{4}, \mathrm{C}_{5}$, or $\mathrm{C}_{6}$ as the experimental configuration, the system generates 127 predictions per second. All the configurations that use the MixNet family as classification engine are less efficient than the ones of the EfficientNet family. In particular, MixNet-XL is the least efficient configuration, returning only 83 predictions within a second.

Answer to $\mathbf{R Q}_{1}$ : EfficientNet and MixNet can predict Covid-19 from CXR images, obtaining a high accuracy and precision. Nevertheless, the MixNet family suffers a low timing efficiency. Among others, EfficientNet-B3 yields the best prediction performance, while maintaining a reasonable recognition speed.

4.2. $R Q_{2}$ : Which transfer learning technique is beneficial to the final outcome? In this research question, we performed experiments following the five-fold cross-validation methodology. Moreover, to further investigate the applicability of the proposed approach, we made use of the $\mathbf{D}_{2}$ dataset, which contains more images than $\mathbf{D}_{1}$ (cf. Table 1). Figure 4(a), Fig. 4(b), and Fig. 4(c) depict the confusion matrices for EfficientNet-B0 using the three different transfer learning techniques mentioned in Section 2.3. The computed metrics for all the confusion matrices are shown in Table 5 . 


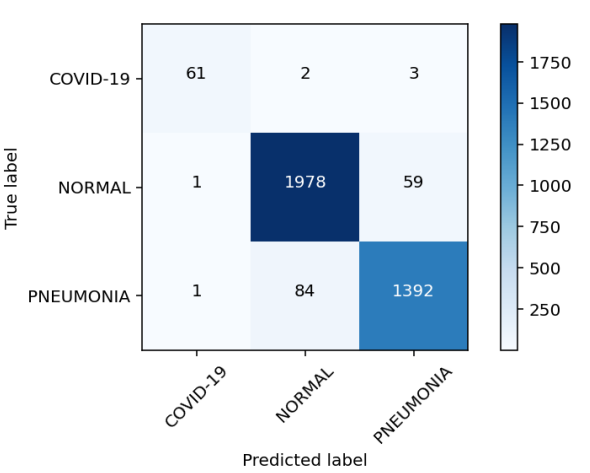

(a) $\mathrm{C}_{1}$

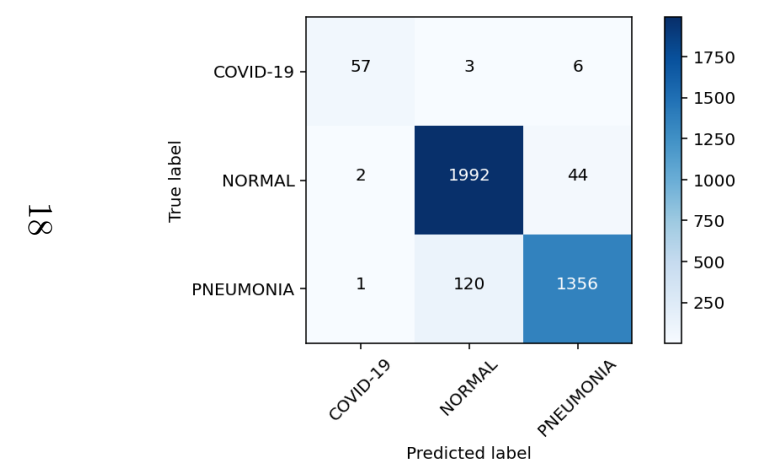

(d) $\mathrm{C}_{4}$

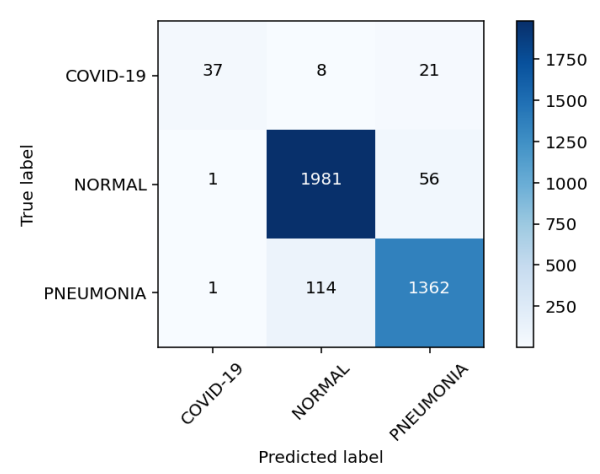

(b) $\mathrm{C}_{2}$

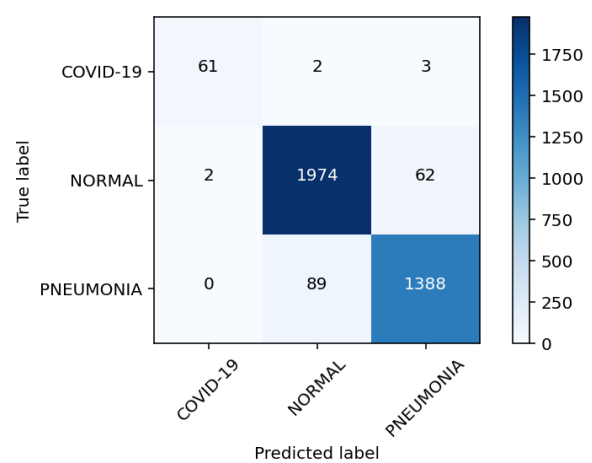

(e) $\mathrm{C}_{5}$

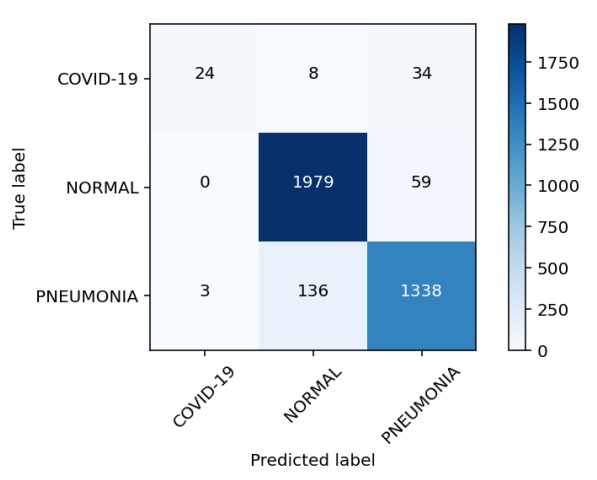

(c) $\mathrm{C}_{3}$

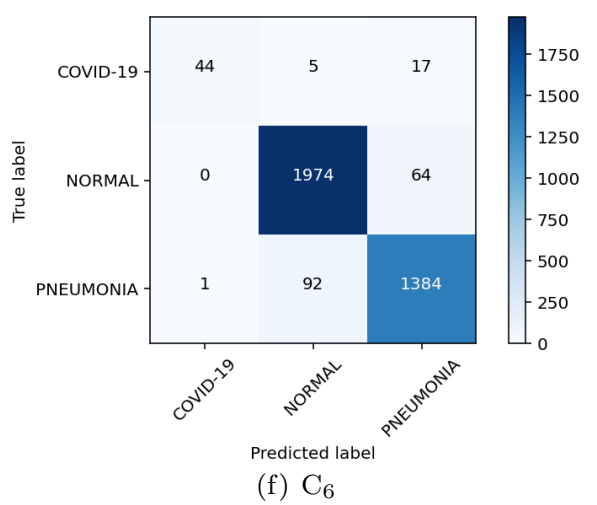

Figure 4: Confusion matrices of EfficientNet-B0 and EfficientNet-B3 using different transfer learning techniques. 


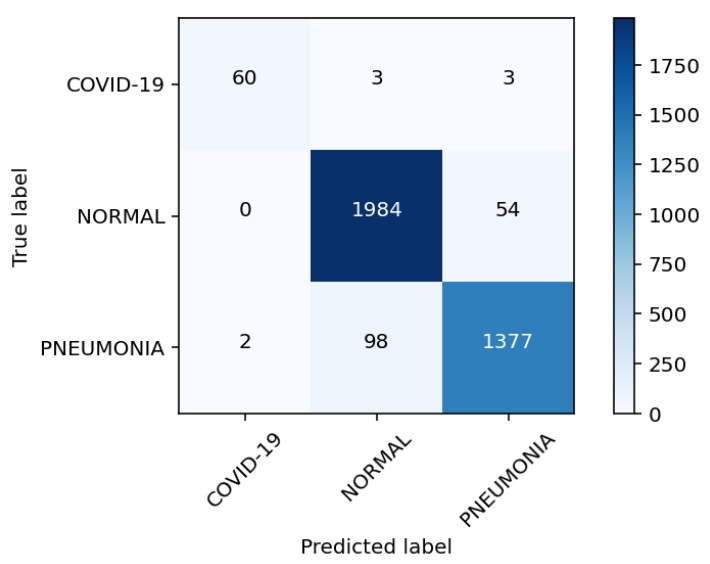

Figure 5: Confusion matrix of MixNet-XL using weights pre-trained with ImageNet $\left(\mathrm{C}_{10}\right)$.

As it can be checked, each transfer learning method may have different effects on the different categories. For instance, training EfficientNet-B0 with weights pre-trained by ImageNet is beneficial to categories Covid-19 and Pneumonia, but not to Normal. In particular, as shown in Fig. 4(a), 61 out of 66 images in Covid-19 are correctly classified, while for Pneumonia 1392 out of 1,477. However, for the Normal category, only 1978 images are correctly classified over a total of 2,038 images, accounting for $97.05 \%$. On the other hand, transfer learning with AdvProp (cf. Fig. 4(b)) induces a better performance for Category Normal, i.e., 1,981 among 2,038 images are classified to the correct categories. Looking at Fig. 4(c), we see that compared to the other transfer learning methods, NS has an adverse effect on the recognition of all the 35 categories. In summary, we can conclude that EfficientNet-B0 with ImageNet transfer learning fosters the best prediction performance.

For EfficientNet-B3, we see that weights pre-trained with ImageNet are beneficial to the Normal category (cf. Fig. 4(d)). At the same time, AdvProp is the transfer learning method that is suitable for recognition of Pneumonia, i.e., 340 it helps to detect 1,388 out of 1,477 pneumonia images, which is best among the others. 
Finally, let us consider the results obtained by running MixNet-XL with weights from ImageNet, as depicted in Fig. 5. The figure shows that MixNetXL does not outperform the other configurations with EfficientNet-B0 and EfficientNet-B3. While it obtains a considerably good performance with Category Normal, correctly classifying 1,984 images among 2,038 images, it suffers of a low precision and recall for the other categories. For instance, with Pneumonia, only 1,377 out of 1,477 images are properly recognized with MixNet-XL together with weights pre-trained with ImageNet.

To sum up, the experiment results demonstrate that, depending on the network family, each transfer learning technique may have a different impact on the final outcome. Taking all metrics in consideration as shown in Table 5, we see that Configuration $\mathrm{C}_{1}$, i.e., the row marked with the gray color, corresponding to training EfficientNet-B0 with weights by ImageNet, is the most effective configuration with respect to accuracy, precision, recall, and $F_{1}$ for almost all categories. Moreover, together with the results obtained from $\mathbf{R Q}_{1}$, we conclude that ImageNet is the best transfer learning strategy for both network families on the two datasets $\mathbf{D}_{1}$ and $\mathbf{D}_{2}$.

Answer to $\mathbf{R Q}_{2}$ : Using EfficientNet-B0 in combination with weights pre-trained from the ImageNet dataset brings the best performance.

\subsection{Threats to Validity}

This section describes the threats to the internal, external, construct, and conclusion validity.

Internal validity. This is related to the internal factors that could have a negative impact on the final outcomes. A possible threat here could come from the results for the Covid-19 category, since they are obtained with a considerably low number of items for training and testing, i.e., $\mathbf{D}_{1}$ with 98 and 10 images and $\mathbf{D}_{2}$ with 327 and 98 images for training and testing, respectively. This threat is mitigated by the other two categories in the datasets, as they contain a considerably large number of items. To the best of our knowledge, there exists 
no dataset with more images for the Covid-19 category. In other words, research in medical imaging on Covid-19 suffers a general lack of data. For this reason, it is unfortunately not possible to test our approach on a larger scale.

External validity. The main threat to external validity is due to the factors that might hamper the generalizability of our results to other scenarios outside the scope of this work, e.g., in practice we may encounter a limited amount of training data. We moderated this threat by evaluating EfficientNet and MixNet using the experimental settings following the five-fold cross-validation methodology. In this way, the original data is split to five parts and only four of them are used to train the system.

3во Construction validity. This is related to the experimental settings presented in the paper, concerning the simulation performed to evaluate the system. To mitigate the threat, the evaluation has been conducted on a training set and a test set, attempting to simulate a real usage where training data is already available for feeding the system, while testing data is the part that needs to be predicted.

Conclusion validity. This concerns all the remaining factors that might have an impact on the obtained outcome. On this respect, the evaluation metrics accuracy, precision, recall, $\mathrm{F}_{1}$ and execution time might cause a related threat. To face the issue, we adopted such measures as recommended by the previous scientific literature related to our setting, and employed the same metrics for evaluating all the classifiers.

\section{Related Work}

Alongside scientists in other disciplines, researchers in Computer Science and Artificial Intelligence reacted quickly to the pandemic. As a matter of fact, in recent months there has been a large number of papers related to the topic Covid-19 and Machine Learning, and multiple Covid-19/ ML applications have been proposed. Table 6 summarizes some of the most notable studies with respect to the number of images for each category as well as prediction accuracy. 
Since in this work we want to support the identification of the disease from CXR images, in the remainder of this section we focus on analyzing these studies.

\begin{tabular}{|l|l|l|l|l|l|}
\hline \multirow{2}{*}{ Study } & \multicolumn{3}{|c|}{ Number of images } & \multirow{2}{*}{ Network } & \multirow{2}{*}{ Acc. (\%) } \\
\cline { 2 - 4 } & Covid-19 & Normal & Pneumonia & \\
\hline \hline Ghoshal et al. $[33]$ & 68 & 1583 & 2786 & ResNet & 89.82 \\
\hline Abbas et al. $[34]$ & 105 & 80 & 11 & $\begin{array}{l}\text { DeTraC based } \\
\text { on ResNet-18 }\end{array}$ & 95.12 \\
\hline Nari et al. $[35]$ & 50 & 50 & - & ResNet-50 & 98.00 \\
\hline $\begin{array}{l}\text { Apostolopous } \\
\text { et al. }[36]\end{array}$ & 224 & 504 & 700 & VGG19 & 93.48 \\
\hline Luz et al. $[37]$ & 183 & - & - & EfficientNet-B3 & 93.90 \\
\hline Zhang et al. $[38]$ & 100 & 1431 & 1531 & ResNet18 & 96.00 \\
\hline Hemdan et al. $[39]$ & 25 & 25 & - & $\begin{array}{l}\text { VGG19, } \\
\text { DenseNet121 }\end{array}$ & 90.00 \\
\hline
\end{tabular}

Table 6: A summary of related studies.

Two studies [40,41] use deep learning to predict which current antivirals might be more effective in patients with coronavirus. Yan et al. [42] propose a specific model to predict if a patient infected with Covid-19 would survive based on his personal data and other risk factors. Other applications have been proposed, like the work by Jiang et al. [43], that identifies the combinations of clinical characteristics of Covid-19 that predict outcomes, and develop a tool with AI capabilities for identifying patients at risk of a more severe impact of the disease.

X-ray machines provide images for quick diagnosis and multiple papers have shown the usefulness of CXR exams in detecting Covid-19 [44]. The work by Hall et al. [45] analyzed 135 chest X-rays confirmed as Covid-19 and 320 chest $\mathrm{X}$-rays of viral and bacterial pneumonia. A pre-trained deep convolutional neural network using Resnet50, was tuned on 102 Covid-19 cases and 102 other pneumonia cases using the ten-fold cross-validation methodology, showing an overall accuracy of $89.2 \%$ with a Covid-19 true positive rate of 0.8039 and an area under the curve (AUC) of 0.95. Still, the dataset used in the work of Hall et al. [45] is considerably small, and thus it is not clear if the approach is able to obtain such a good performance for a larger amount of data. 
The model proposed by Ozturk et al. [46] provides accurate diagnostics for

with X-ray imaging can be used to extract important biomarkers related to the Covid-19 disease. The accuracy obtained in this approach is $96.78 \%, 98.66 \%$, and $96.46 \%$, respectively. Nevertheless, like the work by Narin et al. [35], again the approach has been experimented using a small amount of data, and we ${ }_{445}$ suppose that such a good performance might not possibly be held with larger datasets.

COVID-Net [47] is a deep convolutional neural network design tailored for the detection of Covid-19 cases from CXR images. COVID-Net achieves an 
accuracy of $93.3 \%$, with $98.9 \%$ positive predictive values that is related to the detection of false positives.

A deep learning model has been proposed [48] to detect Covid-19 and differentiate it from common acquired pneumonia and other lung diseases. The analyzed dataset consists of 4,356 chest CT exams collected from 3,322 patients. The per-exam sensitivity and specificity for detecting COVID-19 in the independent test set was 114 of $127(90.0 \%)$ and 294 of 307 (96.0\%), respectively, with an area under the receiver operating characteristic curve (AUC) of 0.96 (p-value $<0.001$ ). The per-exam sensitivity and specificity for detecting community acquired pneumonia in the independent test set was 87\% (152 of 175) and $92 \%$ (239 of 259), respectively.

The classification of medical images has also been covered by the work of Abbas et al. [34]. A CNN, called Decompose, Transfer, and Compose (DeTraC), for the classification of Covid-19 CXR images has been used and an accuracy of $95 \%$ was achieved in the detection of Covid-19 CXR images from normal, and severe acute respiratory syndrome cases. COVID-CAPS [49] is a capsule Network-based Framework for Identification of Covid-19 cases from CXR Images. The approach yielded a good accuracy when working with small datasets.

Ghoshal et al. [50] investigated how drop-weights based Bayesian Convolutional Neural Networks (BCNN) can estimate uncertainty to improve the diagnostic performance of the approaches using publicly available Covid-19 CXR datasets and show that the uncertainty in prediction is highly correlated with the accuracy of prediction.

To the best of our knowledge, compared to different existing studies [34, 36,44 ], our work is the first one that deals with big datasets. In particular, in dataset $\mathbf{D}_{1}$ there are 15,000 images, and in $\mathbf{D}_{2}$ 17,905. However, given 475 such a large amount of data, our proposed approach is still able to obtain a high prediction accuracy, gaining a reasonable recognition speed. Thus, in our opinion the results demonstrate a more reliable applicability in practice, even if it is our belief that the proposed system can be refined with more training data, so as to make it more and more effective in real-world settings. 


\section{Conclusions}

In this paper we proposed a practical solution for the detection of Covid-19 from chest X-ray images exploiting two suitable building blocks: EfficientNet and MixNet as the prediction engine and effective transfer learning strategies. The approach has been validated on two existing datasets which have been widely used in various studies. The experimental results show that our proposed approach outperforms some well-established baselines in terms of prediction performance. For future work, we plan to evaluate and refine our approach by considering additional datasets and tuning other deep neural network configurations.

\section{Acknowledgements}

This work has been supported by (i) the CROSSMINER Project, EU Horizon 2020 Research and Innovation Programme, grant agreement No. 732223; and (ii) the INCIPICT Project, Italian Ministry of Economy and Finance, Cipe resolution n. 135/2012. and anticoagulation, Blood, The Journal of the American Society of Hematology 135 (23) (2020) 2033-2040.

[2] Q. Sun, H. Qiu, M. Huang, Y. Yang, Lower mortality of covid-19 by early recognition and intervention: experience from jiangsu province, Annals of intensive care 10 (1) (2020) 1-4.

[3] F. Jiang, Y. Jiang, H. Zhi, Y. Dong, H. Li, S. Ma, Y. Wang, Q. Dong, H. Shen, Y. Wang, Artificial intelligence in healthcare: past, present and future, BMJ 2 (2017) svn-2017. doi:10.1136/svn-2017-000101.

[4] I. Portugal, P. Alencar, D. Cowan, The use of machine learning algorithms in recommender systems:

A systematic review, Expert Systems with Applications 97 (2018) 205 - 227. doi:https: 
//doi.org/10.1016/j.eswa.2017.12.020.

URL http://www.sciencedirect.com/science/article/pii/ S0957417417308333

[5] P. Domingos, A few useful things to know about machine learning, Commun. ACM 55 (10) (2012) 78-87. doi:10.1145/2347736.2347755.

[6] T. Wuest, D. Weimer, C. Irgens, K.-D. Thoben, Machine learning in manufacturing: advantages, challenges, and applications, Production \& Manufacturing Research 4 (1) (2016) 23-45. doi:10.1080/21693277.2016. 1192517.

[7] D. Sontag, K. Collins-Thompson, P. N. Bennett, R. W. White, S. Dumais, B. Billerbeck, Probabilistic models for personalizing web search, in: Procs. of the Fifth ACM Int. Conf. on Web Search and Data Mining, WSDM '12, ACM, New York, NY, USA, 2012, pp. 433-442. doi:10.1145/2124295. 2124348.

[8] O. Araque, I. Corcuera-Platas, J. F. Sánchez-Rada, C. A. Iglesias, Enhancing deep learning sentiment analysis with ensemble techniques in social applications, Expert Systems with Applications 77 (2017) 236 - 246. doi:https://doi.org/10.1016/j . eswa.2017.02.002.

URL http://www.sciencedirect.com/science/article/pii/ S0957417417300751

[9] X. Mei, H.-C. Lee, K.-y. Diao, M. Huang, B. Lin, C. Liu, Z. Xie, Y. Ma, P. Robson, M. Chung, A. Bernheim, V. Mani, C. Calcagno, K. Li, S. Li, H. Shan, J. Lv, T. Zhao, J. Xia, Y. Yang, Artificial intelligence-enabled rapid diagnosis of patients with covid-19, Nature Medicine (2020) 1-5doi: $10.1038 / s 41591-020-0931-3$.

[10] A. I. Khan, J. L. Shah, M. M. Bhat, Coronet: A deep neural network for detection and diagnosis of covid-19 from chest x-ray images, Computer Methods and Programs in Biomedicine 196 (2020) 105581. 
doi:https://doi.org/10.1016/j.cmpb.2020.105581.

URL http://www.sciencedirect.com/science/article/pii/ S0169260720314140

[11] R. M. Pereira, D. Bertolini, L. O. Teixeira, C. N. Silla, Y. M. Costa, Covid-19 identification in chest x-ray images on flat and hierarchical classification scenarios, Computer Methods and Programs in Biomedicine (2020) 105532doi: https://doi . org/10 . 1016/j . cmpb . 2020 . 105532.

URL http://WwW.sciencedirect.com/science/article/pii/ S0169260720309664

[12] M. Tan, Q. Le, EfficientNet: Rethinking model scaling for convolutional neural networks, in: K. Chaudhuri, R. Salakhutdinov (Eds.), Proceedings of the 36th International Conference on Machine Learning, Vol. 97 of Proceedings of Machine Learning Research, PMLR, Long Beach, California, USA, 2019, pp. 6105-6114.

URL http://proceedings.mlr.press/v97/tan19a.html

[13] M. Tan, Q. V. Le, MixConv: Mixed Depthwise Convolutional Kernels, CoRR abs/1907.09595. arXiv: 1907.09595.

URL http://arxiv.org/abs/1907.09595

[14] O. Russakovsky, J. Deng, H. Su, J. Krause, S. Satheesh, S. Ma, Z. Huang, A. Karpathy, A. Khosla, M. Bernstein, A. C. Berg, L. Fei-Fei, ImageNet Large Scale Visual Recognition Challenge, Int. J. Comput. Vision 115 (3) (2015) 211-252. doi:10.1007/s11263-015-0816-y.

[15] C. Xie, M. Tan, B. Gong, J. Wang, A. Yuille, Q. V. Le, Adversarial Examples Improve Image Recognition, arXiv e-prints (2019) arXiv:1911.09665arXiv : 1911.09665.

[16] Q. Xie, E. Hovy, M.-T. Luong, Q. V. Le, Self-training with noisy student improves imagenet classification, cite arxiv:1911.04252 (2019).

URL http://arxiv.org/abs/1911.04252 
[17] W. Rawat, Z. Wang, Deep convolutional neural networks for image classification: A comprehensive review, Neural Comput. 29 (9) (2017) 2352-2449. doi:10.1162/neco_a_00990.

URL https://doi.org/10.1162/neco_a_00990

[18] N. Srivastava, G. Hinton, A. Krizhevsky, I. Sutskever, R. Salakhutdinov, Dropout: A simple way to prevent neural networks from overfitting, J. Mach. Learn. Res. 15 (1) (2014) 1929-1958.

[19] F. Chollet, Xception: Deep Learning with Depthwise Separable Convolutions, cite arxiv:1610.02357 (2016).

URL http: //arxiv.org/abs/1610.02357

[20] A. G. Howard, M. Zhu, B. Chen, D. Kalenichenko, W. Wang, T. Weyand, M. Andreetto, H. Adam, MobileNets: Efficient Convolutional Neural Networks for Mobile Vision Applications, cite arxiv:1704.04861 (2017).

URL http://arxiv.org/abs/1704.04861

[21] M. Tan, B. Chen, R. Pang, V. Vasudevan, Q. V. Le, MnasNet: PlatformAware Neural Architecture Search for Mobile, CoRR abs/1807.11626. arXiv: 1807.11626. URL http://arxiv.org/abs/1807.11626

[22] H. Cai, L. Zhu, S. Han, ProxylessNAS: Direct neural architecture search on target task and hardware, in: International Conference on Learning Representations, 2019.

URL https: //openreview. net/forum?id=HylVB3AqYm

[23] M. Sandler, A. G. Howard, M. Zhu, A. Zhmoginov, L. Chen, Inverted Residuals and Linear Bottlenecks: Mobile Networks for Classification, Detection and Segmentation, CoRR abs/1801.04381. arXiv: 1801.04381.

URL http://arxiv.org/abs/1801.04381

[24] H. Li, A. Kadav, I. Durdanovic, H. Samet, H. P. Graf, Pruning Filters for Efficient ConvNets, in: 5th International Conference on Learning Rep- 
resentations, ICLR 2017, Toulon, France, April 24-26, 2017, Conference Track Proceedings, 2017.

URL https://openreview. net/forum?id=rJqFGTslg

[25] A. Kamilaris, F. X. Prenafeta-Boldú, Deep learning in agriculture: A survey, Computers and Electronics in Agriculture 147 (2018) 70 - 90. doi:https://doi.org/10.1016/j.compag.2018.02.016.

[26] K. Weiss, T. Khoshgoftaar, D. Wang, A survey of transfer learning, Journal of Big Data 3. doi: 10.1186/s40537-016-0043-6.

[27] L. Torrey, T. Walker, J. Shavlik, R. Maclin, Using advice to transfer knowledge acquired in one reinforcement learning task to another, in: Proceedings of the 16th European Conference on Machine Learning, ECML'05, Springer-Verlag, Berlin, Heidelberg, 2005, pp. 412-424. doi:10.1007/11564096_40.

URL http://dx.doi.org/10.1007/11564096_40

[28] Z. Huang, Z. Pan, B. Lei, Transfer Learning with Deep Convolutional Neural Network for SAR Target Classification with Limited Labeled Data, Remote Sensing 9 (9). doi:10.3390/rs9090907.

[29] L. T. Duong, P. T. Nguyen, C. Di Sipio, D. Di Ruscio, Automated fruit recognition using efficientnet and mixnet, Computers and Electronics in Agriculture 171 (2020) 105326. doi:https: //doi.org/10.1016/j. compag.2020.105326.

URL http://www.sciencedirect.com/science/article/pii/ S0168169919319787

[30] Z. Q. L. Linda Wang, A. Wong, Covid-net: A tailored deep convolutional neural network design for detection of covid-19 cases from chest radiography images (2020). arXiv:2003.09871. 
[31] J. P. Cohen, P. Morrison, L. Dao, Covid-19 image data collection, arXiv 2003.11597.

URL https://github.com/ieee8023/covid-chestxray-dataset

[35] A. Narin, C. Kaya, Z. Pamuk, Automatic detection of coronavirus disease (covid-19) using x-ray images and deep convolutional neural networks (2020). arXiv: 2003 . 10849.

[36] I. D. Apostolopoulos, T. A. Mpesiana, Covid-19: automatic detection from 635

[38] J. Zhang, Y. Xie, Y. Li, C. Shen, Y. Xia, Covid-19 screening on chest Xray images using deep learning based anomaly detection, arXiv preprint arXiv:2003.12338. 
[39] E. E.-D. Hemdan, M. A. Shouman, M. E. Karar, Covidx-net: A framework of deep learning classifiers to diagnose covid-19 in x-ray images, arXiv preprint arXiv:2003.11055.

[40] H. Zhang, K. M. Saravanan, Y. Yang, M. T. Hossain, J. Li, X. Ren, Y. Pan, Y. Wei, Deep learning based drug screening for novel coronavirus 2019ncov, Interdisciplinary Sciences, Computational Life Sciences (2020) 1.

[41] B. R. Beck, B. Shin, Y. Choi, S. Park, K. Kang, Predicting commercially available antiviral drugs that may act on the novel coronavirus (sars-cov-2) through a drug-target interaction deep learning model, Computational and structural biotechnology journal.

[42] L. Yan, H.-T. Zhang, Y. Xiao, M. Wang, C. Sun, J. Liang, S. Li, M. Zhang, Y. Guo, Y. Xiao, et al., Prediction of criticality in patients with severe covid-19 infection using three clinical features: a machine learning-based prognostic model with clinical data in wuhan, MedRxiv.

[43] X. Jiang, M. Coffee, A. Bari, J. Wang, X. Jiang, J. Huang, J. Shi, J. Dai, J. Cai, T. Zhang, et al., Towards an artificial intelligence framework for data-driven prediction of coronavirus clinical severity, CMC: Computers, Materials \& Continua 63 (2020) 537-51.

[44] L. Brunese, F. Mercaldo, A. Reginelli, A. Santone, Explainable deep learning for pulmonary disease and coronavirus covid-19 detection from x-rays, Computer Methods and Programs in Biomedicine 196 (2020) 105608. doi:https://doi .org/10.1016/j . cmpb.2020. 105608. S0169260720314413

[45] L. O. Hall, R. Paul, D. B. Goldgof, G. M. Goldgof, Finding covid-19 from chest x-rays using deep learning on a small dataset, arXiv preprint arXiv:2004.02060. 

Acharya, Automated detection of covid-19 cases using deep neural networks with x-ray images, Computers in Biology and Medicine (2020) 103792.

[47] L. Wang, A. Wong, Covid-net: A tailored deep convolutional neural network design for detection of covid-19 cases from chest x-ray images, arXiv preprint arXiv:2003.09871.

[48] L. Li, L. Qin, Z. Xu, Y. Yin, X. Wang, B. Kong, J. Bai, Y. Lu, Z. Fang, Q. Song, et al., Artificial intelligence distinguishes covid-19 from community acquired pneumonia on chest ct, Radiology (2020) 200905.

[49] P. Afshar, S. Heidarian, F. Naderkhani, A. Oikonomou, K. N. Plataniotis, A. Mohammadi, Covid-caps: A capsule network-based framework for identification of covid-19 cases from x-ray images, arXiv preprint arXiv:2004.02696.

[50] B. Ghoshal, A. Tucker, Estimating uncertainty and interpretability in deep learning for coronavirus (covid-19) detection, arXiv preprint arXiv:2003.10769. 